\title{
Collision-induced luminescence from the impact of ions and cluster ions on solid rare gases
}

\author{
Christoph Sieber ${ }^{\text {a }}$ Wolfgang Harbich ${ }^{\text {a }}$ Karl-Heinz Meiwes-Broer ${ }^{\mathrm{b}}$ Christian Félix ${ }^{\mathrm{a}, *}$ \\ ${ }^{a}$ Institut de Physique des Nanostructures, École Polytechnique Fédérale de Lausanne (EPFL), CH-1015 Lausanne, Switzerland \\ ${ }^{\mathrm{b}}$ Fachbereich Physik, Universität Rostock, D-18051 Rostock, Germany
}

\begin{abstract}
Light emission during the collision of ions and cluster ions on a metal surface covered by rare gas matrices of variable thickness is reported. The light intensity as a function of distance from the metal surface follows the classical image dipole theory. The spectral signature of the light corresponds to the fluorescence of the neutral cluster species deposited as well as to fragments. The nature of the processes involved in this luminescence, in the neutralization and the excitation are discussed. A microscopic model for the mechanism is proposed: in this model rare gas excitons are produced in the collision process.
\end{abstract}

Key words: Metal clusters, luminescence, fluorescence, collisions, rare gas matrices, excitons PACS: 36.40.Mr, 36.40.Vz, 61.46.+w

\section{Introduction}

Energetic particle surface interaction results in the excitation of the surface and particles electronic system. This excitation can result in electron and photon emission or simply be absorbed by the substrate. In the first two cases, information can be gained on the particle substrate electronic system. More specifically in ion surface collisions charge transfer processes from the substrate to the ionized projectile become an important energy relaxation channel. In particular in low energy deposition as will be described below, the charge transfer channel considerably contributes to the energy relaxation mechanism.

Mass selected cluster deposition can be considered as a special case of ion surface scattering. Mass selection prior to deposition necessarily implies the

\footnotetext{
* Corresponding author. Fax: +4121 6933603

Email address: christian.felix@epfl.ch (Christian Félix).
}

particle to be charged before mass filtering and the cluster is then deposited on either a conducting substrate which supplies the neutralizing electron or codeposited with electrons on insulating substrates. This technique has attracted increasing attention during the last years due to its strength to produce tailored nanostructures. One method to prepare intact clusters at surfaces utilizes slowing down in rare gas spacer layers to provide soft landing. Micron thick rare gas spacer layers are employed in the so called matrix isolation technique where the specie of interest is embedded. This allows an accumulation of very high target densities for subsequent investigations. The idea is that the inert host does only slightly change the electronic structure of the cluster.

This work focuses on light emission during ion and cluster ion impact. When the ions collide with the bare conducting surface, radiative processes are very weak and predicted to be in the range of $10^{-9}$ photons/ion [1]. The reason for this low yield is es- 
sentially the very effective energy transfer mechanism for a radiative dipole in the vicinity of a metallic substrate. However when introducing an argon spacer layer, dipole radiation becomes possible. Prerequisite is the supply with neutralizing electrons, which have to come from the metal $(\mathrm{Au})$ substrate.

After a short description of the experimental setup we will present and discuss the results on the total photon yield as a function of spacer thickness. The second part focuses on the spectral information contained in the emitted light which will be shown to be material and size specific. Throughout the paper we will employ the term luminescence for the collision induced light.

\section{Experimental setup}

The overall setup is described elsewhere [2]. Metal clusters are produced by sputtering from a metal target, extracted by an ion lens system and mass selected by a quadrupole mass filter. The mass selected cluster ions are deflected by $90^{\circ}$ with an electrostatic quadrupole, that acts as a filter for the neutral silver particles as well as an energy filter for the cluster ions. The positively charged clusters are focused by a second lens system on a cold $(\mathrm{T}<$ $25 \mathrm{~K}$ ) gold plated copper plate, where they are codeposited with the Ar to form a seeded matrix. The argon flux towards the cold surface is set in order to achieve an Ar to cluster ratio of typically $10^{4}: 1$. No external neutralization electrons are provided to the ions. The sample is electrically isolated and by setting its potential, the deposition energy of the clusters can be varied and the deposition current measured. The deposition energy ranges from a few eV to several hundred $\mathrm{eV}$, with a particle current of a few $\mathrm{nA}$. The light is collected during the deposition by an optical fiber $(\phi=400 \mu \mathrm{m})$ located close to the cluster deposition spot. The light is then analyzed by an optical spectrometer coupled to a liquid nitrogen cooled CCD detector for spectra acquisitions, or to a photomultiplier tube for time resolved experiments. The spectra are not corrected for the transmission and efficiency of the different optical elements (optical fiber, spectrometer, CCD).

\section{Luminescence properties}

\subsection{Luminescence intensity versus spacer thickness}

Fig. 1 shows the integral photon yield during the deposition of $\mathrm{Ag}_{2}^{+}$as a function of matrix thickness which is, at constant gas flux, equivalent to time. The photon yield is normalized to the cluster current, also shown. No light is observed for the bare metal surface. As the spacer layer grows, luminescence is recorded versus layer thickness, starting at $\sim 20 \mathrm{~nm}$ and saturating after $\sim 200 \mathrm{~nm}$. When a thick matrix is grown first then the luminescence starts immediately and is linear with the incident cluster current as shown in Fig. 1c.

It is well known [3] that the presence of a metal surface influences the luminescence by the simple fact that a dipole interacts with its own radiation reflected by the surface at larger distance and by nonradiative energy dissipation at smaller distances. The problem has been studied for example by Chance et al. [4], where they were interested in the lifetime of an excited molecule near a partially reflecting metal mirror. The signal intensity depends on the orientation of the dipole relative to the metal surface, on the distance to the surface, on the dielectric functions of the metal and matrix, and on the emission wavelength.

The result of such a calculation for an emission wavelength of $470 \mathrm{~nm}$ is shown in Fig. $1 \mathrm{~b}$. The wavelength chosen corresponds to the main emission yield for $\mathrm{Ag}_{2}^{+}$. Since the light is collected parallel to the metal surface the signal is averaged between signal resulting from a dipole radiation parallel and perpendicular to the surface. This is reasonable since the clusters deposited in the matrix have a random orientation. Note that there are no adjustable parameters in this calculation. The agreement between this simulation and the measurements is quite remarkable and fully explains the evolution of the luminescence intensity with the matrix thickness.

\subsection{Luminescence temperature dependence}

We observe (Fig. 2) a roughly linear decrease in intensity with increasing matrix temperature, being quenched for Ar at $32 \mathrm{~K}$. This is probably related to the crystallinity of the matrix, which improves with increasing growth temperatures, implying that the luminescence process requires a matrix with struc- 

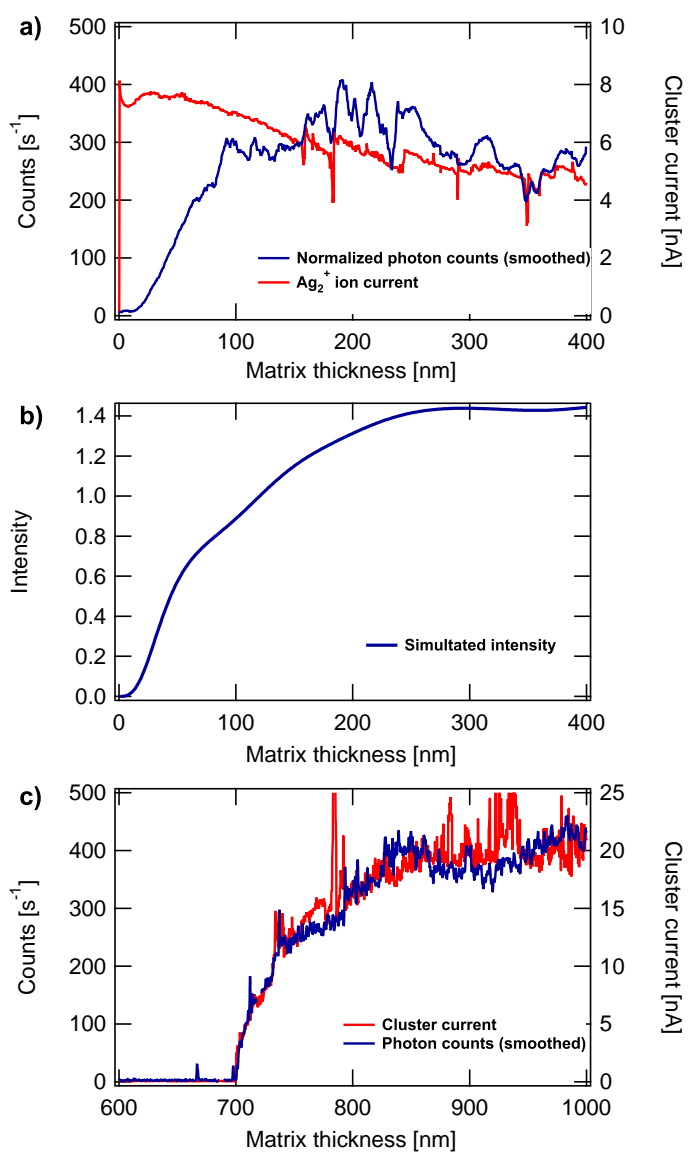

Fig. 1. Evolution of light emitted by $\mathrm{Ag}_{2}^{+}$collisions measured at a matrix temperature of $20 \mathrm{~K}$. a) The deposition starts with no argon matrix on the metal surface the clusters are deposited together with argon to form a seeded matrix. No light emission is observed at zero coverage, the luminescence starts with a matrix thickness of $\sim 20 \mathrm{~nm}$ and saturates after $\sim 200 \mathrm{~nm}$. The photon counts have been corrected for current variation. b) Simulated intensity of the emission at $470 \mathrm{~nm}$ parallel to a gold surface (see text). c) The matrix is first built without clusters until a thickness of $700 \mathrm{~nm}$ and then the clusters are directed towards the matrix together with argon to form a seeded matrix. In this case the emitted light is directly proportional to the cluster current. For technical reasons, the cluster current is slowly increased over time.

tural defects.

\subsection{Luminescence properties versus cluster material and size}

The spectral signature of the luminescence resulting from the impact of $\mathrm{Ag}_{1}^{+}, \mathrm{Au}_{1}^{+}$and $\mathrm{Cu}_{1}^{+}$in argon is shown in Fig. 3. The luminescence is compared to laser induced fluorescence measurement performed

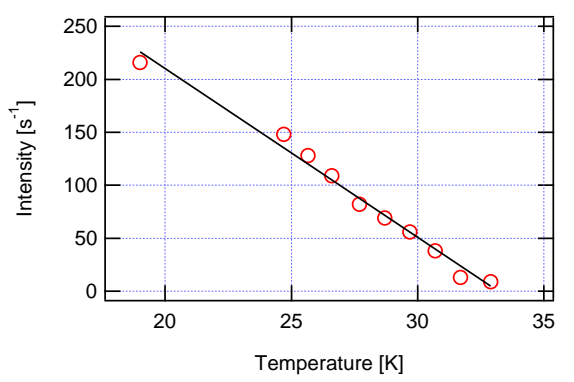

Fig. 2. Luminescence intensity versus matrix temperature during the collision of $\mathrm{Ag}_{2}^{+}$. At $34 \mathrm{~K}$ the evaporation of the matrix sets in. The experiment is conducted under steady argon gas deposition and with a deposition energy for $\mathrm{Ag}_{2}^{+}$ of $30 \mathrm{eV}$. The line is a guide to the eye.

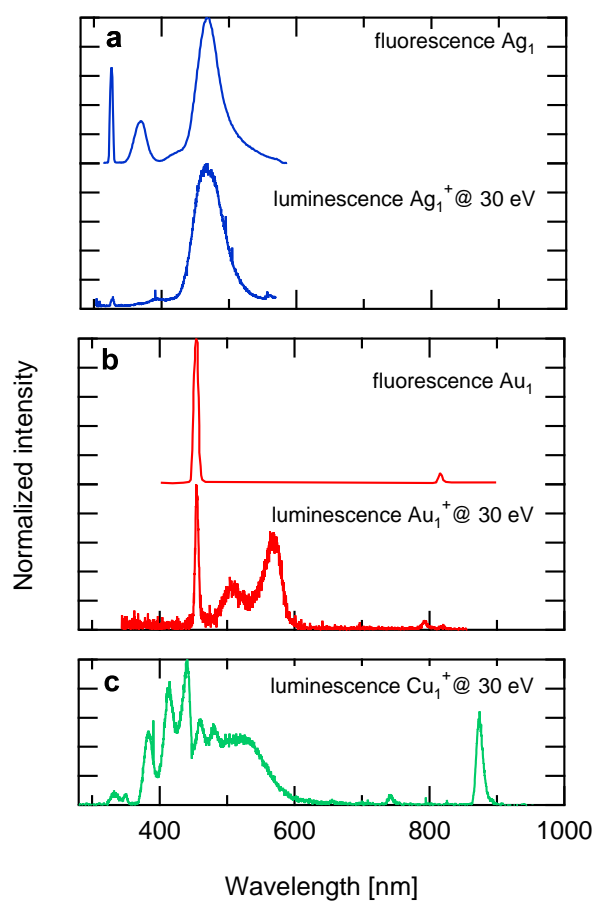

Fig. 3. (a,b)Spectrally resolved luminescence from $\mathrm{Ag}_{1}^{+}$and $\mathrm{Au}_{1}^{+}$collisions and laser induced fluorescence spectra of $\mathrm{Ag}_{1}$ and $\mathrm{Au}_{1}$ in argon for comparison. (c) Luminescence spectrum from $\mathrm{Cu}^{+}$collisions.

on the same system; for fluorescence measurements the mass-selected clusters are deposited with an excess of low energy electrons that allow for the cluster neutralization. The clear distinction between the different luminescence spectra, and moreover identical positions of the peaks when compared to the laser induced fluorescence of the corresponding neutral particles embedded in argon proves that this luminescence originates from the neutralized particle in the collision process. The luminescence of the 


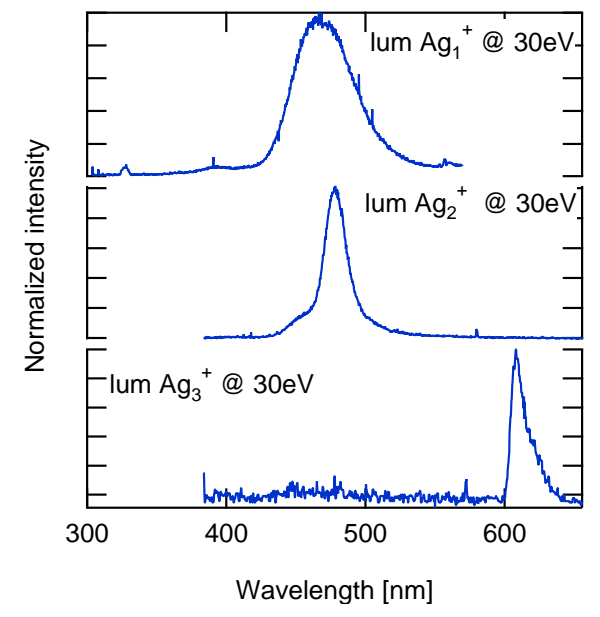

Fig. 4. Spectrally resolved luminescence of $\mathrm{Ag}_{2}^{+}$and $\mathrm{Ag}_{3}^{+}$ during the deposition in an argon matrix.

neutral atom is observed despite the fact that the cation is deposited. It is therefore clear that either the fluorescence happens after the neutralization process or that it is a direct result of the neutralization, where the neutralizing electron radiates down to the ground state. A tentative discussion on the neutralization process is given at the end of this paper. Notice that the relative intensities between the peaks change significantly between the fluorescence and the luminescence, and that additional peaks are present in the luminescence of $\mathrm{Au}$.

Since the luminescence signal reflects the elemental nature of the colliding ions it can be expected that the cluster size information should be as well contained in the spectra. This is indeed the case as shown in Fig. 4. Luminescence during the collision of silver cluster cations $(n=2,3)$ is spectrally resolved and the peaks positions are in excellent agreement with laser induced fluorescence of the corresponding neutral species in argon [5]. We tried to measure the luminescence spectra of larger particles as well. Although laser induced fluorescence has been observed in our group for $\mathrm{Ag}_{4}, \mathrm{Ag}_{8}$ and $\mathrm{Ag}_{9}$ [6-8], no evidence for collision-induced luminescence has been found. This is probably related to the excitation mechanism of the luminescence.

From the laser-induced fluorescence, it is known that the spectra exhibit matrix-dependent shifts $[9,5]$. Thus the agreement with the fluorescence hints at that the emitting particle has sufficient time to find a stable environment. This statement is further supported by the observation of fragmentation channels. Increasing the deposition energy results in an increase of the luminescence of the fragments and a simultaneous reduction of the luminescence of the incoming cluster. This proves that the luminescence process does not happen during but after the impact, for the cluster has the time to fragment and to capture an electron before emitting a photon.

\section{Analysis of the luminescence process}

The neutralization/excitation process which is responsible for the observed luminescence is complex and will be discussed only briefly, tracing the essential ideas. A more complete analysis together with further experimental data which support our model will be given in a forthcoming publication.

The current measurements shown in Fig. 1 give the number of electrons that flow towards the sample to neutralize the positive charges of the clusters. It is well known that excess electrons in rare gas matrices are almost unbound and can move very easily through a liquid or a solid rare gas [10] until they find a structural defect that can trap them or an ion that they can neutralize. The electrons flowing through the matrix are a result of the collision of the cluster ions, since the measured current falls to zero when the cluster beam is stopped. The question of the source of energy $(\sim 5 \mathrm{eV})$ allowing an electron to escape the work function of the metal to be injected into the matrix remains, moreover the mechanism responsible for the observed luminescence is not yet understood.

The key in the interpretation of our observation is the production of excitons in the cluster rare gas collision. Excitons and energy transfer mechanisms between excitons and impurities (the metal atoms and clusters in this case) have been studied thoroughly [11-20]. In these studies, the excitons have been excited either by x-ray absorption, resonant excitation with synchrotron light or electron bombardment. Exciton excitation by cluster ion collisions has, to our knowledge, not been studied.

We compare our results to experiments by Schrimpf et al. [15], that performed fluorescence measurements by exciting with monochromatized light from a synchrotron storage ring, being able to excite either resonantly the metal atoms, by inner shell excitations of the metal atoms or by producing excitons in the rare gas matrix. They show that the energy transfer from excitons to the metallic impurities leads to fluorescence of the impurities in the host matrix. First the relative peak inten- 
sities in the luminescence of $\mathrm{Ag}_{1}$ (Fig. 3) is very similar to their observations for inner-shell and exciton excitations. The presence of additional peaks in the luminescence of $\mathrm{Au}_{1}$ in $\mathrm{Ar}$ is very similar to the additional peak at $2.45 \mathrm{eV}$ that they observe for $\mathrm{Au}_{1}$ in $\mathrm{Kr}$ and tentatively attributed to $\mathrm{Au}-\mathrm{H}$ complexes in that paper. Finally the temperature dependence of the luminescence (Fig. 2) is in good agreement with their observation in Kr matrices. The comparison provides experimental evidence that the excited states from which the luminescence proceeds is identical in both experiments. While this is not yet a definitive proof that excitons are generated in the collision process, it shows that it is a reasonable assumption.

Once produced, free excitons (FE) can diffuse through the solid, and transfer energy according to the fate of the FE. FE can undergo radiative recombination, be trapped either at impurities and induce the luminescence of this impurity [15], or be self-trapped in the solid leading to atomic or molecular self-trapped exciton emissions. Notice that all the exciton decay mechanisms can produce photons of sufficient energy to induce photoelectrons on the metal substrate, therefore explaining the mechanism responsible for the neutralization of the cations.

Energy can be transferred from the matrix surface to the clusters respectively to the metal substrate by the diffusion of $\mathrm{FE}[14,16]$ during the lifetime $10^{-12} \mathrm{~s}$ of the FE. The diffusion length of $\mathrm{FE}$ depends strongly on the matrix preparation, being very short (about $20 \mathrm{~nm}$ ) in polycrystalline matrices and extending to several hundreds $\mathrm{nm}$ in well annealed matrices at low temperature [16]. This could explain the observed temperature dependence of the luminescence (Fig. 2).

\section{Conclusion}

Luminescence of ions and small metal clusters ions impinging on rare gas covered metal surfaces has been observed. The luminescence intensity close to the metal surface is effectively quenched and the intensity profile can be understood by the simple fact that a dipole interacts with its own radiation reflected by the surface at larger distance and by nonradiative energy dissipation at smaller distances. The luminescence signature is very close to the fluorescence signature of the neutral particle and is size and material specific. A model is proposed that im- plies the creation of excitons during the impact and a trapping of the exciton on structural defects of the rare gas solid.

This work has been supported by the Swiss National Science Foundation.

\section{References}

[1] N. Lorente, R. Monreal, M. Alducin, and P. Apell, Z. Phys. D 41, 143 (1997).

[2] F. Conus, J. T. Lau, V. Rodrigues, and C. Félix, accepted in Rev. Sci. Instrum. (2006).

[3] D. H. Waldeck, A. P. Alivisatos, and C. B. Harris, Surf. Sci. 158, 103 (1985).

[4] R. R. Chance, A. Prock, and R. Silbey, Adv. Chem. Phys. 37, 1 (1978).

[5] S. Fedrigo, W. Harbich, and J. Buttet, J. Chem. Phys. 99, 5712 (1993).

[6] C. Félix, C. Sieber, W. Harbich, J. Buttet, I. Rabin, W. Schulze, and G. Ertl, Chem. Phys. Lett. 313, 105 (1999).

[7] C. Félix, C. Sieber, W. Harbich, J. Buttet, I. Rabin, W. Schulze, and G. Ertl, Phys. Rev. Lett. 86, 2992 (2001).

[8] C. Sieber, W. Harbich, J. Buttet, C. Félix, R. Mitrić, and V. Bonačić-Koutecký, Phys. Rev. A 70, 041201 (2004).

[9] W. Harbich, S. Fedrigo, and J. Buttet, Z. Phys. D 26, 138 (1993).

[10] E. B. Gordon, V. V. Khmelenko, and O. S. Rzhevsky, Chem. Phys. Lett. 217, 605 (1994).

[11] N. Schwentner, E.-E. Koch, and J. Jortner, Electronic excitations in condensed rare gases, Vol. 107 of Springer tracts in modern physic (Springer, Berlin, 1985).

[12] G. Zimmerer, in Excited-State Spectroscopy in Solids (Holland Pub. Co., Amsterdam, 1987), pp. 37-110.

[13] K. S. Song and R. T. Williams, Self-Trapped Excitons, Vol. 105 of Springer Series in Solid State Science (Springer, Berlin, 1996).

[14] Z. Ophir, N. Schwentner, B. Raz, M. Skibowski, and J. Jortner, J. Chem. Phys. 63, 1072 (1975).

[15] A. Schrimpf, B. Herkert, L. Manceron, U. Schriever, and H.-J. Stöckmann, Phys. Stat. Sol. B 165, 469 (1991).

[16] B. Herkert, A. Schrimpf, K. Göttsche, T. Bornemann, and H.-J. Stöckmann, Phys. Rev. B 51, 15763 (1995).

[17] M. Lengen, M. Joppien, R. von Pietrowski, and T. Möller, Chem. Phys. Lett. 229, 362 (1994).

[18] A. Schrimpf, C. Boekstiegel, H.-J. Stöckmann, T. Bornemann, K. Ibbeken, J. Kraft, and B. Herkert, J. Phys. Cond. Matter 8, 3677 (1996).

[19] J. Becker, O. N. Grigorashchenko, A. N. Ogurtsov, M. Runne, E. V. Savchenko, and G. Zimmerer, J. Phys. D 31, 749 (1998).

[20] E. V. Savchenko, O. N. Grigorashchenko, A. N. Ogurtsov, V. V. Rudenkov, G. B. Gumenchuk, M. Lorenz, A. M. Smith-Gicklhorn, M. Frankowski, and V. E. Bondybey, Surf. Rev. Lett. 9, 353 (2002). 